\title{
A Brief Review on Multimedia-Based Health Education Applications: Current Trend and Future Potential
}

\author{
Lua Pei Lin ${ }^{1}$, Nor Khaira Wahida Khairuzzaman ${ }^{2}$ \\ ${ }^{1}$ Institute for Community Development \& Quality of Life (i-CODE), Universiti Sultan Zainal Abidin, Gong \\ Badak Campus, 21300 Kuala Terengganu, and ${ }^{2}$ Faculty of Medicine \& Health Sciences, Universiti Sultan \\ Zainal Abidin, Kota Campus, 20400 Kuala Terengganu, Terengganu, Malaysia.
}

\section{ARTICLE INFO}

Received : :01/09/2014

Accepted : : : : $\quad: 01 / 10 / 2014$

Published : :01/12/2014

\section{KEYWORD}

Multimedia

Health Education

Patients

Caregivers

\section{ABSTRACT}

Objective: This paper intended to review and analyse relevant published articles which have studied or applied multimedia as the educational medium for patients or their caregivers. The benefits were also recorded. Method: The search was performed across the databases EBSCO Host, Springer Link, Science Direct and PubMed for relevant studies. Only fulltext articles using English as a language of publication were included. Eligible articles included any usage of multimedia intervention as health information delivery for patients or caregivers. No restriction for publication date was set to permit a wider capture. Result: Twenty articles met the inclusion criteria resulting in the involvement of a total of 5,760 respondents. The studies have been conducted in various countries mostly in the North American region followed by Europe. The focused disease for each study varied from asthma to cognitive impairment but most were on cancer. Problems in caregiving and depression were also reported. The overall data suggested that the multimedia-based education had generated modest improvement in self-efficacy, patient satisfaction, coping skills, and perceptions of social support. Cost benefits were also recorded. Additionally, patients' behavioural changes were well maintained in parallel with the intervention programme. Conclusion: The evolution of multimedia as an educational medium is growing and its incorporation has benefited health education management especially in improving patients' and their family's psychosocial outcomes. However, due to still limited scientific evidence to support its value, further multimedia-based interventions should be developed out of the need to share information and knowledge among patients as well as caregivers.

(C) Medical Education Department, School of Medical Sciences, Universiti Sains Malaysia. All rights reserved.

CORRESPONDING AUTHOR: Pei Lin LUA, Head, Community Health Research Cluster, Institute for Community Development and Quality of Life (i-CODE), Faculty of Medicine and Health Sciences, Universiti Sultan Zainal Abidin (UniSZA), Gong Badak Campus, 21300 Kuala Terengganu, Terengganu, MALAYSIA Email: peilinlua@unisza.edu.my / peilinlua@hotmail.com 


\section{Introduction}

The term multimedia can be defined as the components characterized by the presence of text, picture, sound, animation and video which is organized in combination of some or all components into a coherent programme [1]. To date, multimedia evolutions clearly indicate positive benefits in health education [2] especially in theory-based programmes which showed improvement in self-management practices on both patients and caregivers. These programmes disciplined the patients particularly on their daily medication intake as well as help in stress management and depression [3].

Thus far, important effects of education on health at every level of social aggregation are not limited to providing sources of health-related information but also function to raise the recipients' motivation, skills and confidence (self-efficacy) necessary to take action to improve health. [4] The main key in managing a chronic condition is to activate self-management, as patients and their families spend most of their time outside the healthcare system [5]. Therefore, they must learn the skills and techniques to live with their chronic condition to navigate their disease management and improve quality of life (QoL) [6]. Computer-based simulations provide realistic environment to present interactive learning experience to the patients or caregivers. The technology provides focused groups of subject the opportunities to repeat and deliberately practice the knowledge with structured guidance [3], hence ensuring information sustainability.

Many recent innovations have been created for the roles of knowledge delivery in various education fields and this includes in healthcare [7]. To meet the demands of the 21st century, researchers have begun to investigate the framework of health behaviours and its influential factors among patients and their caregivers in order to develop interactive programmes as educational medium [8]. Technology-based intervention can offer potentially strong support in the areas of prevention and detection as well as managing patients' life $[9,10]$.

Outcomes from previous research have revealed positive evidence from the behavioural, educational and social sciences perspectives and indicated that among others, knowledge and attitude towards a particular disease are the keys to achieve independence for care activities [11]. It is also not surprising that modern technologies have the potential to indirectly improve patients or caregivers' QoL through health education because better understanding will improved disease management. The goals could be achieved by providing the necessary skills and information to deal with daily obstacles they face in managing the disease condition [11].

Most technology interventions place focus on psycho-educational outcomes of patients or their caregivers by addressing symptom management, psychosocial support and resource identification. Positive effects can be seen in the reduction of recipients' depression due to increased self-efficacy in managing the condition [12, 13]. Certain studies which provide guidance of behavioural disturbances management appeared to decrease recipients' anger and hostility as well as improved sense of self-control [14]. It is also essential to demonstrate improvement of patient outcomes in providing high-quality of care but simultaneously reducing the conflicts faced by caregivers in fulfilling the demand to prepare adequate home care support [15].

Limited reviews are currently available to support health learning enhancement through multimedia technology. Thus, this paper intends to review and analyse the relevant published articles which have studied or applied multimedia as an educational medium for patients as well as caregivers. Through this, the intervention programme of each study will be identified to examine its multimedia incorporation and the subsequent outcomes of field tests conducted. 


\section{Method}

\section{Search strategy and selection criteria}

An electronic search was conducted using the EBSCO Host, Springer Link, Science Direct and PubMed databases for relevant articles. The search was initiated using various combinations of the following keywords: 'multimedia', 'health education', ‘technology', ‘chronic disease', 'patients', ‘caregivers', 'healthcare' and 'telemedicine'. The eligible articles were restricted to full-text English articles, with focus only in journal articles. Information on studies which have applied multimedia in health information dissemination as intervention was the major aim. The recipients of each study were also limited to only patients and/or family caregivers. The search criteria did not include any limitation on publication date, and the earliest eligible article was published in 2002. The reference lists of included articles were also searched. Irrelevant and excluded articles were mostly abstracts, special topic, review articles or that the respondents comprised healthcare professionals or the general public.

\section{Data collection and analysis}

The titles and abstracts of the identified articles were screened to eliminate duplicates and unrelated articles. A manual full text review was performed on all articles meeting the inclusion criteria in order to extract information from each article. The extracted information is presented in Tables 1 and 2 which include socio-demographic characteristics and scientific evidence on multimedia applications.

Article information and respondent demographic distributions were summarized according to year / author(s), country, study objective(s), disease / problem, age, gender, ethnicity / race and education level. Additionally, multimedia applications were also identified and analysed according to study design / study setting, sample size / respondents, intervention programme, instruments / measurements and final findings.

For the outcome comparisons, we focused primarily on psychosocial outcomes which could lead to improvement in QoL, stress reduction and increase in self-efficacy of patients and caregivers. Besides, it can provide suggestions on the proper strategies to overcome patients' burden and caregivers' problems in caregiving. In fact, the main objective is to set a global focus on existing multimedia applications as educational medium used to deliver health information and knowledge for both patients and caregivers. In addition, this review also intended to document the benefits of multimedia-based health education for further investigations.

\section{Result}

\section{General Overview}

The combined electronic database search resulted in 1,797 articles. Most articles were excluded due to the nature of their studies which focused on professional caregivers or public respondents. After final selection, a total of 20 relevant studies met the inclusion criteria. Sociodemographic characteristics of the respondents are detailed in Table 1. The collective scientific evidence of multimedia applications for health education is shown Table 2. A wide range of multimedia learning for various chronic diseases was reported from 2002 to 2013. Almost all articles were published in different journals except for the Journal of Medical Systems and Journal of Community Health which published two articles each [16-19].

Most articles had originated from the United States of America (USA) [17, 19, 20-25]. It was obvious that most studies were conducted in developed countries since information was rather lacking from developing and poorer countries. The total sample in these 20 studies consisted of 5,760 respondents, with the majority being patients $(n=17)$ and the education programmes often presented via computer softwares $(n=6)$ [16-19, 21, 22, 24-34].

\section{Socio-demographic characteristics}

Eight out of 20 studies were conducted in Western countries, especially in the USA. Among the 20 studies, five evaluated the outcomes of multimedia health education programme for cancer patients and/or caregivers [19-21, 24, 28]. Studies on asthma and diabetes 
patients have been identified through three articles each [17, 18, 22, 23, 26, 33]. Another two studies focused on chronic diseases in general, while one study each was on cardiovascular disease, scoliosis and cognitive impairment [16, 27, 33, 29, 34]. There was also a report on care giving problem and depression [25, 35]. Due to the various diseases covered, this review included a wide range of respondents, aged from 10 years old to 78 years old, inclusive of children, teenagers, adults and the elderly.

Across all studies, seven did not report on gender distributions. The remaining 13 studies which have a total respondent of 3,196 showed a rather well-balanced distribution between male and female proportions. However, an article which reported on a breast cancer study involved a huge number of female patients $(n=1,197)$ [19], reflected by the final sample at $74.2 \%$ compared to males at $25.4 \%$.

According to Table 1, the ethnicities in each country were represented mainly by their largest group of population i.e. USA dominated by the Whites while Taiwan have mostly Chinese respondents [20, 29]. Additionally, the educational level of respondents in half of the articles was not reported. Nevertheless, most education levels reported were based on the respective country's educational system and no specific education requirement was mentioned in any article for study inclusion.

\section{Scientific Investigations on Multimedia Applications}

\section{Study design and sample size}

Most studies were based on randomized controlled design $(\mathrm{n}=14)$, four had constructed cross-sectional studies while the other two were exploratory in nature - Table 2. The crosssectional study was conducted qualitatively only to access the information needed without specific investigation on the related applications. The respondents recruited in all 20 studies included a combination of patients-caregivers $(n=2)$, or patients only $(n=17)$, or caregivers only $(n=1)$, with a total respondents of 5,760. Among these, only two articles did not report the sample size of respondents. The biggest recruitment number was reported in a cancer study with a total of 2,134 patients, whereas the lowest sample size involved only six respondents with cognitive disabilities.

\section{Multimedia applications / appliances}

In view of the multimedia applications, most studies delivered their educational programmes through computer software, which were presented as: 1) video footage of instructional film, 2) personnel video instructions, 3) multimedia video curriculum, and 4) multimedia interactive programme. For example, a report on cancer education created its instructional video by filming a workshop given by oncology personnel. The instructional contents included safety precautions, communication, intention and frame of mind, positioning on home furniture, manual techniques for comfort and relaxation and acupressure for pain, anxiety and nausea.

Besides structured computerised programmes, four articles reported on the development of web-based interactions and telematics intervention respectively. Another six studies utilized usage of mobile gadgets and other devices i.e. Nintendo Wii Fit Plus-based and wireless health monitoring system.

\section{Study outcomes}

Despite the different outcome measurements used, the general outcomes seemed to project a common theme in most aspects measured. Through the multimedia-based health education, reported impacts on patients involved: 1) reduction in stress level, 2) increased selfefficacy (in communication and self-care management) and 3) improved physical activity maintenance, health behaviours, knowledge and attitude. In addition, another investigation involving vestibular loss patients reported the increase in patients' enjoyment after receiving rehabilitation through a multimedia programme.

Among the caregivers, several improvements can be seen especially their caregiving satisfaction. Successful reduction in their concern and worries towards patients was also reported. Furthermore, 
they seemed to have experienced increased selfefficacy as well as coping skills on handling caregiving problems, i.e. becoming more prepared to cope with anticipated problem.

Improvements in both patients' and caregivers' health-related quality of life (HRQoL) level were also demonstrated. Moreover, at least two studies showed enhancements in patients' social support and increased sense of trust between patients, caregivers and physicians. Thus, the implementation of multimedia applications indirectly uncovered and maintained social support needs as well as providing health information.

Additionally, healthcare services costs have also been reduced in the studies involving asthma, cancer and diabetes patients. A study on asthma showed that patients provided with multimediabased health information were permitted better opportunities to exchange information with health providers at home regarding the disease condition.[23] Patients eventually managed to save on travelling cost for commuting between home and hospital.[28]

\section{Instruments / Measurements}

Almost all studies used different instruments to assess the application outcomes. Explicably, the tools differed from one another due to the various diseases investigated and the variety of respondent groups (patients / caregivers). The utilized instruments were chosen or designed respectively according to the article purposes but mostly assessed stress level, depression, HRQoL, knowledge and attitude.

For example, studies implementing web-based programmes assessed the outcome parameters using either: 1) electronic contact record form (ECRF) or web-login record, 2) telephone audiorecord or 3) website analysis and measurement inventory (WAMMI). Stress in a cancer study was assessed via Perceived Stress Scale (PSS10) for both caregivers and patients. Whilst studies which measured QoL used: 1) Malay Quality of Life in Epilepsy-30 (MQOLIE-30), 2) Mini Asthma Related Quality of Life Questionnaire (Mini AQLQ), and Functional
Assessment of Cancer Therapy-General (FACT$\mathrm{G}$, version 4) for epilepsy, asthma, and cancer, respectively. Other miscellaneous instruments included: 1) breast cancer knowledge, 2) Vestibular Rehabilitation Benefits Questionnaire (VRBQ), and 3) Diabetes Self-Care Activities Measure (SDSCA) plus Spoken Knowledge in Low Literacy in Diabetes scale (SKILL-D). Two studies which measured anxiety and depression both used Hospital Anxiety and Depression Scale (HADS). 
Table 1: Socio-demographic characteristics of the research participants according to publication year.

\begin{tabular}{|c|c|c|c|c|c|c|c|c|}
\hline No. & $\begin{array}{l}\text { Year / Author } \\
\text { (s) }\end{array}$ & Country & Objective (s) & $\begin{array}{l}\text { Disease / } \\
\text { Problem } \\
\text { (n) }\end{array}$ & $\begin{array}{l}\text { Age (years) / Mean } \\
(\%) / \text { Mean }( \pm \text { SD) } / \\
\text { Range }\end{array}$ & Gender $n(\%)$ & Ethnicity $n(\%)$ & Education level $n$ (\%) \\
\hline 1. & $\begin{array}{l}2013 / \\
\text { Collinge } \\
\text { et al. }\end{array}$ & $\begin{array}{l}\text { United } \\
\text { States of } \\
\text { America }\end{array}$ & $\begin{array}{l}\text { To evaluate the outcomes of a } \\
\text { multimedia instructional } \\
\text { programme for family caregivers. }\end{array}$ & $\begin{array}{l}\text { Cancer } \\
\text { (194) }\end{array}$ & 53.1 & $\begin{array}{l}\text { - } \text { Male } \\
=66(34.0) \\
\text { - Female } \\
=128(66.0)\end{array}$ & $\begin{array}{l}\text { - White } \\
=127(65.5) \\
\text { - African American } \\
=26(13.4) \\
\text { - Asian } \\
=22(11.3) \\
\text { - Hispanic } \\
=16(8.2) \\
\text { - Native American } \\
=3(1.5)\end{array}$ & 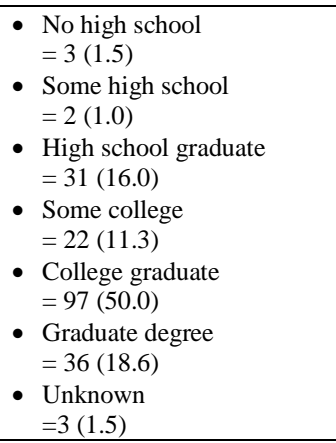 \\
\hline 2. & $\begin{array}{c}2013 / \\
\text { Lua \& Neni }\end{array}$ & Malaysia & $\begin{array}{l}\text { To investigate the impact of } \\
\text { SMS-based education for } \\
\text { epilepsy patients. }\end{array}$ & $\begin{array}{l}\text { Epilepsy } \\
(144)\end{array}$ & $30.5( \pm 11.8)$ & $\begin{array}{l}\text { - Male } \\
=73(50.7) \\
\text { - Female } \\
=71(49.3)\end{array}$ & $\begin{array}{l}\text { - } \text { Malay } \\
=131(91.0) \\
\text { - Chinese } \\
=9(6.2) \\
\text { - Indian } \\
=3(2.1) \\
\text { - Others } \\
=1(0.7) \\
\end{array}$ & $\begin{aligned} \text { - } & \leq \mathrm{SPM} / \text { Cambridge O' level } \\
& =110(76.4) \\
\text { - } & \text { SPM/Cambridge O' level } \\
& =34(23.6)\end{aligned}$ \\
\hline 3. & $\begin{array}{l}2013 \text { Stanton } \\
\text { et al. }\end{array}$ & $\begin{array}{l}\text { United } \\
\text { States of } \\
\text { America }\end{array}$ & $\begin{array}{l}\text { To evaluate the outcomes of } \\
\text { psycho-educational trials for } \\
\text { cancer patients. }\end{array}$ & $\begin{array}{l}\text { Cancer } \\
(2,134)\end{array}$ & 57.5 & N/A & $\begin{array}{l}\text { - } \text { Non-Hispanic White } \\
=1,544(72.4) \\
\text { - African American } \\
=206(9.65) \\
\text { - Other } \\
=58(2.72)\end{array}$ & $\begin{array}{l}\text { - High school or less } \\
=292(13.7) \\
\text { - Some college } \\
=490(23.0) \\
\text { - College graduate / above } \\
=1,083(50.7)\end{array}$ \\
\hline 4. & $\begin{array}{l}2013 / \\
\text { Tancredi } \\
\text { et al. }\end{array}$ & $\begin{array}{l}\text { United } \\
\text { States of } \\
\text { America }\end{array}$ & $\begin{array}{l}\text { To examine the effectiveness of } \\
\text { messages system on depression } \\
\text { care among patients. }\end{array}$ & $\begin{array}{l}\text { Depression } \\
\quad(867)\end{array}$ & 51.8 & 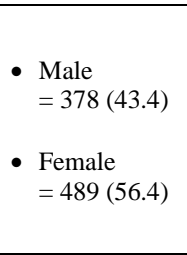 & $\begin{array}{l}\text { - } \text { Hispanic/Latino } \\
=123(14.2) \\
\text { - } \text { Black/Non-Hispanic } \\
=168(19.4) \\
\text { - Asian / Other } \\
=90(10.4) \\
\text { - White } \\
=486(56.1)\end{array}$ & $\begin{array}{l}\text { - College / graduate degree } \\
=391(45.1)\end{array}$ \\
\hline
\end{tabular}

* N/A = Not available

SMS = Short messages service 


\begin{tabular}{|c|c|c|c|c|c|c|c|c|}
\hline No. & $\begin{array}{l}\text { Year / Author } \\
\text { (s) }\end{array}$ & Country & Objective (s) & $\begin{array}{c}\text { Disease / } \\
\text { Problem } \\
\text { (n) }\end{array}$ & $\begin{array}{c}\text { Age (years) / Mean } \\
(\%) / \text { Mean }( \pm S D) / \\
\text { Range }\end{array}$ & Gender $n(\%)$ & Ethnicity $n(\%)$ & Education level $n(\%)$ \\
\hline 5. & $\begin{array}{c}2012 \text { / } \\
\text { Antypas \& } \\
\text { Wangberg }\end{array}$ & Norway & $\begin{array}{l}\text { To assess the effects of website } \\
\text { programme on self-management } \\
\text { behaviours maintenance. }\end{array}$ & $\begin{array}{l}\text { Cardio- } \\
\text { vascular } \\
\text { diseases } \\
(255)\end{array}$ & N/A & N/A & N/A & N/A \\
\hline 6. & $\begin{array}{l}2012 / \\
\text { Meldrum } \\
\text { et al. }\end{array}$ & Ireland & $\begin{array}{l}\text { To compare the effectiveness of } \\
\text { rehabilitation treatment between } \\
\text { conventional and virtual method. }\end{array}$ & $\begin{array}{l}\text { Vestibular } \\
\text { loss } \\
(80)\end{array}$ & N/A & N/A & N/A & N/A \\
\hline 7. & $\begin{array}{c}2012 / \\
\text { Spinsante \& } \\
\text { Gambi }\end{array}$ & Italy & $\begin{array}{l}\text { To monitor the health of the } \\
\text { elderly patients. }\end{array}$ & $\begin{array}{l}\text { Chronic } \\
\text { diseases } \\
(15)\end{array}$ & $23-53$ & N/A & N/A & N/A \\
\hline 8. & $\begin{array}{c}2011 / \text { } \\
\text { Ahmed et al. }\end{array}$ & Canada & $\begin{array}{l}\text { To evaluate the acceptability and } \\
\text { efficacy of using My Asthma } \\
\text { Portal (MAP). }\end{array}$ & $\begin{array}{l}\text { Asthma } \\
\text { (80) }\end{array}$ & $18-70$ & N/A & N/A & N/A \\
\hline 9. & $\begin{array}{l}2011 / \\
\text { Khan et al. }\end{array}$ & $\begin{array}{l}\text { United } \\
\text { States of } \\
\text { America }\end{array}$ & $\begin{array}{l}\text { To evaluate the impact of a } \\
\text { computer multimedia diabetes } \\
\text { education programme on self- } \\
\text { patient management. }\end{array}$ & $\begin{array}{c}\text { Diabetes } \\
\text { (129) }\end{array}$ & $51.5( \pm 11.7)$ & $\begin{array}{l}\text { - Male } \\
\quad=74(57.4) \\
\text { - Female } \\
\quad=55(42.6)\end{array}$ & $\begin{array}{l}\text { - Hispanic } \\
=34(26.4) \\
\text { - African American } \\
=63(48.8) \\
\text { - White } \\
=4(3.1) \\
\text { - Asian } \\
=22(17.1) \\
\text { - Other } \\
=3(2.3)\end{array}$ & N/A \\
\hline 10. & $\begin{array}{l}2010 \text { / } \\
\text { Chang et al. }\end{array}$ & Taiwan & $\begin{array}{l}\text { To provide travel guidance } \\
\text { support for persons with cognitive } \\
\text { disabilities. }\end{array}$ & $\begin{array}{l}\text { Cognitive } \\
\text { impairment } \\
\text { (6) }\end{array}$ & $19-76$ & 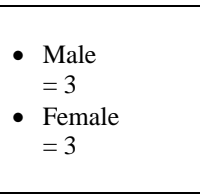 & $\begin{array}{l}\text { - Chinese } \\
=6\end{array}$ & $\begin{array}{l}\text { - College } \\
=1 \\
\text { - High school } \\
=4 \\
\text { - Elementary school } \\
=1\end{array}$ \\
\hline 11. & $\begin{array}{l}2010 / \\
\text { Shing et al. }\end{array}$ & Taiwan & $\begin{array}{l}\text { To evaluate the outcomes of } \\
\text { telecare information platform for } \\
\text { aging population. }\end{array}$ & $\begin{array}{l}\text { - Aging } \\
\text { - Chronic } \\
\text { diseases }\end{array}$ & N/A & N/A & N/A & N/A \\
\hline 12. & $\begin{array}{l}2009 / \\
\text { Mac-Culloch } \\
\text { et al. }\end{array}$ & Canada & $\begin{array}{l}\text { To identify health-specific needs } \\
\text { for scoliosis patients. }\end{array}$ & $\begin{array}{l}\text { Scoliosis } \\
\text { (11) }\end{array}$ & $10-18$ & 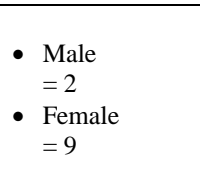 & $\begin{array}{l}\text { - Asian } \\
=1(9.1) \\
\text { - African American } \\
=3(27.3) \\
\text { - Caucasian } \\
=7(63.6)\end{array}$ & N/A \\
\hline
\end{tabular}




\begin{tabular}{|c|c|c|c|c|c|c|c|c|}
\hline No. & $\begin{array}{l}\text { Year/ } \\
\text { Author } \\
\text { (s) }\end{array}$ & Country & Objective (s) & $\begin{array}{l}\text { Disease / } \\
\text { Problem } \\
\text { (n) }\end{array}$ & $\begin{array}{l}\text { Age (years) / Mean } \\
(\%) / \text { Mean }( \pm \text { SD) / } \\
\text { Range }\end{array}$ & Gender $n(\%)$ & Ethnicity n (\%) & Education level $n$ (\%) \\
\hline 13. & $\begin{array}{l}2009 / \\
\text { Sobel et } \\
\text { al. }\end{array}$ & $\begin{array}{l}\text { United } \\
\text { States of } \\
\text { America }\end{array}$ & $\begin{array}{l}\text { To promote asthma self-care } \\
\text { concepts among African } \\
\text { American adults. }\end{array}$ & $\begin{array}{l}\text { Asthma } \\
(130)\end{array}$ & $50.2(15.3)$ & $\begin{array}{l}\text { - Male } \\
=31(23.8) \\
\text { - Female } \\
=99(76.2)\end{array}$ & $\begin{array}{l}\text { - African American } \\
=130(100.0)\end{array}$ & 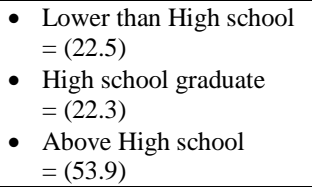 \\
\hline 14. & $\begin{array}{l}2009 / \\
\text { Übeyli }\end{array}$ & Turkey & $\begin{array}{l}\text { To provide information } \\
\text { knowledge in medicine } \\
\text { health care. }\end{array}$ & $\begin{array}{l}\text { Diabetes } \\
(\mathrm{N} / \mathrm{A})\end{array}$ & $10-75$ & N/A & N/A & N/A \\
\hline 15. & $\begin{array}{l}2008 / \\
\text { Mosnaim } \\
\text { et al. }\end{array}$ & $\begin{array}{l}\text { United } \\
\text { States of } \\
\text { America }\end{array}$ & $\begin{array}{l}\text { To evaluate the ability of the } \\
\text { music tracks in increasing asthma } \\
\text { knowledge. }\end{array}$ & $\begin{array}{l}\text { Asthma } \\
\text { (28) }\end{array}$ & 13.4 & $\begin{array}{l}\text { - } \text { Male } \\
=9(60.0) \\
\text { - Female } \\
=6(50.0)\end{array}$ & $\begin{array}{l}\text { - African American = } \\
27(100.0)\end{array}$ & N/A \\
\hline 16. & $\begin{array}{l}2004 / \\
\text { Bielli et } \\
\text { al. }\end{array}$ & Italy & $\begin{array}{l}\text { To develop a new system for self- } \\
\text { reported patient outcomes }\end{array}$ & $\begin{array}{l}\text { Cancer } \\
(97)\end{array}$ & $52(13.9)$ & $\begin{array}{l}\text { - Male } \\
\quad=30(31.0) \\
\text { - Female } \\
=67(69.1)\end{array}$ & N/A & $\begin{array}{l}\text { - Years of education } \\
=10(4.2)\end{array}$ \\
\hline 17. & $\begin{array}{c}2004 / \\
\text { Diefenbac } \\
\text { h \& Butz }\end{array}$ & $\begin{array}{l}\text { United } \\
\text { States of } \\
\text { America }\end{array}$ & $\begin{array}{l}\text { To introduce multimedia } \\
\text { education programme for prostate } \\
\text { cancer patients. }\end{array}$ & $\begin{array}{l}\text { Cancer } \\
\text { (33) }\end{array}$ & $66(8)$ & $\begin{array}{l}\text { - Male } \\
=18(54.5) \\
\text { - Female } \\
=15(45.4)\end{array}$ & $\begin{array}{l}\text { - } \text { Caucasian } \\
=(92.0) \\
\text { - African American } \\
=(6.0) \\
\text { - Hispanic } \\
=(1.0) \\
\text { - Asian/ Pacific } \\
\quad \text { Islander } \\
=(1.0)\end{array}$ & $\begin{array}{l}\text { - Grade school } \\
=(7.0) \\
\text { - High school } \\
=(47.0) \\
\text { - College } \\
=(23.0) \\
\text { - Post-graduate } \\
=(23.0)\end{array}$ \\
\hline 18. & $\begin{array}{l}2003 / \\
\text { Chambers } \\
\text { et al. }\end{array}$ & $\begin{array}{l}\text { United } \\
\text { Kingdom }\end{array}$ & $\begin{array}{l}\text { To provide an application for } \\
\text { carers to cope with emergencies } \\
\text { and caring problems. }\end{array}$ & $\begin{array}{l}\text { Caregiving } \\
\text { (234) }\end{array}$ & $32-78$ & $\begin{array}{l}\text { - } \text { Male } \\
=(24.0) \\
\text { - Female } \\
=(76.0)\end{array}$ & N/A & N/A \\
\hline 19. & $\begin{array}{l}2002 \text { / } \\
\text { Nebel et } \\
\text { al. }\end{array}$ & Germany & $\begin{array}{l}\text { To train and help diabetic patients } \\
\text { on daily food intakes. }\end{array}$ & $\begin{array}{l}\text { Diabetes } \\
\text { (126) }\end{array}$ & $66.3 \pm 4.5$ & $\begin{array}{l}\text { - } \text { Male } \\
=73(58.0) \\
\text { - Female } \\
=53(42.1) \\
\end{array}$ & N/A & N/A \\
\hline 20. & $\begin{array}{l}2002 / \\
\text { Valdez et } \\
\text { al. }\end{array}$ & $\begin{array}{l}\text { United } \\
\text { States of } \\
\text { America }\end{array}$ & $\begin{array}{l}\text { To improve knowledge, attitude } \\
\text { and screening intentions on breast } \\
\text { cancer. }\end{array}$ & $\begin{array}{l}\text { Cancer } \\
(1,197)\end{array}$ & $40-64$ & $\begin{array}{l}\text { - Female } \\
=1,197(100.0)\end{array}$ & $\begin{array}{l}\text { - Latinas } \\
=1,197(100.0)\end{array}$ & $\begin{array}{l}\text { - } 8 \text { years or less } \\
=910(76.0) \\
\text { - } \text { High school } \\
=287(24.0)\end{array}$ \\
\hline
\end{tabular}


Table 2: Scientific evidence on multimedia health education applications according to publication year.

\begin{tabular}{|c|c|c|c|c|c|c|c|}
\hline No. & $\begin{array}{c}\text { Year / } \\
\text { Author(s) }\end{array}$ & $\begin{array}{c}\text { Study Design } \\
\& \\
\text { Study Setting } \\
\end{array}$ & $\begin{array}{c}\text { Sample Size } \\
(n) / \\
\text { Respondent } \\
\text { Type } \\
\end{array}$ & $\begin{array}{l}\text { Multimedia } \\
\text { applications / } \\
\text { appliances } \\
\text { (conditions) } \\
\end{array}$ & $\begin{array}{l}\text { Instruments / } \\
\text { Measurements }\end{array}$ & Psychosocial Outcomes & Comments \\
\hline 1. & $\begin{array}{l}2013 / \\
\text { Collinge } \\
\text { et al. }\end{array}$ & $\begin{array}{l}\text { - Randomized } \\
\text { controlled study } \\
\text { - Nine cancer } \\
\text { treatment centres } \\
\text { and support and } \\
\text { advocacy } \\
\text { organizations in } \\
\text { USA }\end{array}$ & $\begin{array}{l}\text { Total sample } \\
\quad=194 \\
\text { Patients \& } \\
\text { Caregivers }\end{array}$ & $\begin{array}{c}\text { Multilingual } \\
\text { 78-min } \\
\text { Instructional } \\
\text { Digital Versatile } \\
\text { Disc (DVD) } \\
\text { (Cancer) }\end{array}$ & $\begin{array}{l}\text { Caregivers: } \\
\text { - Caregiver Attitudes Towards } \\
\text { Caregiving (investigator-generated } \\
\text { questions) } \\
\text { - Caregiver Reaction Assessment } \\
\text { - Perceived Stress Scale (PSS-10) } \\
\text { Patients: } \\
\text { - Perceived Stress Scale (PSS-10) } \\
\text { - Functional Assessment of Cancer } \\
\text { Therapy-General } \\
\text { version 4) (FACT-G, }\end{array}$ & $\begin{array}{l}\text { Caregiver outcomes: } \\
\text { - High compliance among } \\
\text { caregivers in their weekly } \\
\text { assigned activities } \\
\text { - Satisfaction on the ability to } \\
\text { help patients feel better } \\
\text { - Reduced concern about } \\
\text { causing distress to patients } \\
\text { - Increased self-efficacy to } \\
\text { provide massage for patients } \\
\text { Patient outcomes: } \\
\text { - Improved quality of life } \\
\text { (QoL) } \\
\text { - Reduced stress }\end{array}$ & $\begin{array}{l}\text { Randomization was based } \\
\text { solely on ethnicity, with no } \\
\text { matching by type, stage of } \\
\text { cancer, relationship type or } \\
\text { other variables. }\end{array}$ \\
\hline 2. & $\begin{array}{l}2013 / \\
\text { Lua \& Neni }\end{array}$ & $\begin{array}{l}\text { - Randomized } \\
\text { controlled study } \\
\text { - Three public } \\
\text { hospitals in } \\
\text { Terengganu, } \\
\text { Kelantan and } \\
\text { Pahang } \\
\end{array}$ & $\begin{array}{l}\text { Total sample } \\
\quad=144 \\
\text { Patients }\end{array}$ & $\begin{array}{c}\text { SMS-Based Mobile } \\
\text { Epilepsy Education } \\
\text { System (MEES) } \\
\text { (Epilepsy) }\end{array}$ & $\begin{array}{l}\text { Malay Quality of Life in Epilepsy-30 } \\
\text { (MQOLIE-30) }\end{array}$ & $\begin{array}{l}\text { Patients in intervention group } \\
\text { showed improvement in health- } \\
\text { related quality of life } \\
\text { (HRQoL). }\end{array}$ & $\begin{array}{l}\text { There was no assurance that } \\
\text { the messages were received, } \\
\text { read and understood by the } \\
\text { intended recipients. }\end{array}$ \\
\hline 3. & $\begin{array}{l}2013 / \\
\text { Stanton et } \\
\text { al. }\end{array}$ & $\begin{array}{l}\text { - Randomized } \\
\text { controlled study } \\
\text { - Participants' } \\
\text { home }\end{array}$ & $\begin{array}{l}\text { Total sample } \\
\quad=2,134 \\
\text { Patients }\end{array}$ & $\begin{array}{c}\text { Web-Based } \\
\text { Multimedia } \\
\text { Programme } \\
\text { (Cancer) }\end{array}$ & $\begin{array}{ll}\text { - } & \text { Electronic Contact Record Form } \\
& \text { (ECRF) } \\
\text { - Call frequency data } \\
\text { - Call-to-action email } \\
\end{array}$ & $\begin{array}{l}\text { Increased sense of trust on } \\
\text { cancer information } \\
\text { programmes to facilitate } \\
\text { patients' needs of information. }\end{array}$ & $\begin{array}{l}\text { Respondents' inclusion criteria } \\
\text { were highly restrictive. }\end{array}$ \\
\hline 4. & $\begin{array}{l}2013 / \\
\text { Tancredi et } \\
\text { al. }\end{array}$ & $\begin{array}{ll}\text { - } & \text { Randomized } \\
\text { controlled study } \\
\text { - } & \text { Two primary care } \\
\text { clinics r of } \\
\text { academic medical } \\
\text { centres. } \\
\end{array}$ & $\begin{array}{l}\text { Total sample } \\
\quad=867 \\
\text { Patients }\end{array}$ & $\begin{array}{c}\text { Activating } \\
\text { Messages for } \\
\text { Enhancing Primary } \\
\text { Care Practice } \\
\text { (AMEP2) } \\
\text { (Depression) } \\
\end{array}$ & $\begin{array}{l}\text { - Patient report } \\
\text { - Short telephone interview }\end{array}$ & $\begin{array}{l}\text { Improved self-efficacy for } \\
\text { communication and care } \\
\text { engagement. }\end{array}$ & $\begin{array}{l}\text { The interventions were } \\
\text { recommended for improving } \\
\text { depression detection and its } \\
\text { treatment in primary care. }\end{array}$ \\
\hline
\end{tabular}

* N/A = Not available 


\begin{tabular}{|c|c|c|c|c|c|c|c|}
\hline No. & $\begin{array}{c}\text { Year / } \\
\text { Author(s) }\end{array}$ & $\begin{array}{c}\text { Study Design } \\
\& \\
\text { Study Setting } \\
\end{array}$ & $\begin{array}{c}\text { Sample Size }(n) \\
\text { / Respondent } \\
\text { Type }\end{array}$ & $\begin{array}{l}\text { Multimedia } \\
\text { applications / } \\
\text { appliances } \\
\text { (conditions) } \\
\end{array}$ & $\begin{array}{l}\text { Instruments / } \\
\text { Measurements }\end{array}$ & Psychosocial Outcomes & Comments \\
\hline 5. & $\begin{array}{l}2012 / \\
\text { Antypas \& } \\
\text { Wangberg }\end{array}$ & $\begin{array}{l}\text { - } \text { Cluster } \\
\text { randomized } \\
\text { study. } \\
\text { - Skibotn } \\
\text { Rehabilitation } \\
\text { Centre. }\end{array}$ & $\begin{array}{l}\text { Total sample } \\
\quad=255 \\
\text { Patients }\end{array}$ & $\begin{array}{l}\text { - Website-based } \\
\text { (E- } \\
\text { rehabilitation) } \\
\text { - Mobile text } \\
\text { messages } \\
\text { (Cardiovascular } \\
\quad \text { diseases) }\end{array}$ & $\begin{array}{ll} & \text { Web-login } \\
\text { - } & \text { WHO Rose questionnaire. } \\
\text { - } & \text { International Physical Activity } \\
& \text { Questionnaire (IPAQ). } \\
\text { - } & \text { Perceived competence for regular } \\
& \text { physical exercise (PC-EX). } \\
\text { - } \quad \text { Regulatory focus questionnaire. } \\
\quad \text { Hospital anxiety and depression scale } \\
\\
\text { (HADS). } \\
\text { Euroqol (EQ-5D). } \\
\end{array}$ & $\begin{array}{l}\text { Increased the chance of } \\
\text { physical activity } \\
\text { maintenance. }\end{array}$ & $\begin{array}{l}\text { The applications and } \\
\text { instructions on the website } \\
\text { were simple and easy to } \\
\text { understand for senior citizens } \\
\text { who were not familiar with the } \\
\text { internet. }\end{array}$ \\
\hline 6. & $\begin{array}{l}2012 / \\
\text { Meldrum } \\
\text { et al. }\end{array}$ & $\begin{array}{l}\text { - Randomized } \\
\text { controlled study } \\
\text { - Two Dublin } \\
\text { universities } \\
\text { teaching hospital }\end{array}$ & $\begin{array}{l}\text { Total sample } \\
\quad=80 \\
\text { Patients }\end{array}$ & $\begin{array}{l}\text { Nintendo Wii Fit } \\
\text { Plus-based } \\
\text { (NWFP) vestibular } \\
\text { rehabilitation } \\
\text { (Vestibular loss) }\end{array}$ & $\begin{array}{l}\text { - Three dimensional gait analysis (3DGA) } \\
\text { - Dynamic gait index (DGI) } \\
\text { - Computerised dynamic posturography } \\
\text { (Equitest-NeuroCom) } \\
\text { - Vestibular rehabilitation benefits } \\
\text { questionnaire (VRBQ) } \\
\text { - Dynamic visual acuity (DVA) } \\
\text { - Hospital anxiety and depression scale } \\
\text { (HADS) } \\
\text { - System usability questionnaire }\end{array}$ & $\begin{array}{lr}\text { Enjoyment, challenge and } \\
\text { enriched } & \text { sensory } \\
\text { environment } & \text { during } \\
\text { rehabilitation } & \text { were } \\
\text { reported. } & \end{array}$ & $\begin{array}{l}\text { The application set a standard } \\
\text { procedure for rehabilitation } \\
\text { which made it easy for the } \\
\text { patients to undergo } \\
\text { rehabilitation. }\end{array}$ \\
\hline 7. & $\begin{array}{l}2012 \text { / } \\
\text { Spinsante \& } \\
\text { Gambi }\end{array}$ & $\begin{array}{l}\text { - Randomized } \\
\text { controlled study } \\
\text { - Participants' } \\
\text { home }\end{array}$ & $\begin{array}{l}\text { Total sample } \\
\quad=15 \\
\text { Patients }\end{array}$ & $\begin{array}{l}\text { TV-based } \\
\text { healthcare service } \\
\text { (t-health) } \\
\text { (Chronic diseases) }\end{array}$ & $\begin{array}{l}\text { - Oxymeter } \\
\text { - Bracelet } \\
\text { - Glycaemia meter } \\
\text { - Breathing tester } \\
\text { - Wireless network hub }\end{array}$ & $\begin{array}{l}\text { - The application was } \\
\text { stable from technical } \\
\text { error } \\
\text { - The return channel } \\
\text { interface was correctly } \\
\text { performed } \\
\text { - Patients clearly } \\
\text { understood the } \\
\text { contents' functions }\end{array}$ & $\begin{array}{l}\text { The system was not designed } \\
\text { to be used in emergency } \\
\text { conditions, but only for long- } \\
\text { term monitoring of patients at } \\
\text { home. }\end{array}$ \\
\hline 8. & $\begin{array}{l}2011 \text { / } \\
\text { Ahmed et al. }\end{array}$ & $\begin{array}{l}\text { - Randomized } \\
\text { controlled study } \\
\text { - Pulmonary } \\
\text { clinics in two } \\
\text { tertiary care } \\
\text { hospitals in } \\
\text { Montreal }\end{array}$ & $\begin{array}{l}\text { Total sample } \\
\quad=80 \\
\text { Patients }\end{array}$ & $\begin{array}{l}\text { My Asthma Portal } \\
\text { (MAP) } \\
\text { (Asthma) }\end{array}$ & $\begin{array}{l}\text { - Mini asthma related quality of life } \\
\text { questionnaire (Mini AQLQ) } \\
\text { - Technology acceptance model (TAM) } \\
\text { - Amount of fast acting bronchodilators } \\
\text { (beta-2 agonists) usage } \\
\text { - Chronic disease self-efficacy scale } \\
\text { - Quebec provincial health database } \\
\text { (RAMQ) }\end{array}$ & $\begin{array}{l}\text { - Potential rin } \\
\text { providing support } \\
\text { tools for health } \\
\text { behaviour varied } \\
\text { among patients } \\
\text { Cost reduction in } \\
\text { care provision }\end{array}$ & $\begin{array}{l}\text { Respondents were among } \\
\text { younger and educated patients. } \\
\text { Thus, the result might be } \\
\text { rather biased. }\end{array}$ \\
\hline
\end{tabular}




\begin{tabular}{|c|c|c|c|c|c|c|c|}
\hline No. & $\begin{array}{c}\text { Year / } \\
\text { Author(s) }\end{array}$ & $\begin{array}{c}\text { Study Design } \\
\& \\
\text { Study Setting }\end{array}$ & $\begin{array}{c}\text { Sample Size } \\
\text { (n)/ } \\
\text { Respondent } \\
\text { Type }\end{array}$ & $\begin{array}{l}\text { Multimedia } \\
\text { applications / } \\
\text { appliances } \\
\text { (conditions) } \\
\end{array}$ & $\begin{array}{l}\text { Instruments / } \\
\text { Measurements }\end{array}$ & Psychosocial Outcomes & Comments \\
\hline 9. & $\begin{array}{l}2011 / \\
\text { Khan et al. }\end{array}$ & $\begin{array}{l}\text { - Randomized } \\
\text { controlled study } \\
\text { - County clinic in } \\
\text { Chicago }\end{array}$ & $\begin{array}{l}\text { Total sample } \\
\quad=129 \\
\text { Patients }\end{array}$ & $\begin{array}{l}\text { Living Well With } \\
\text { Diabetes Computer } \\
\text { Multimedia } \\
\text { Program } \\
\text { (Diabetes) }\end{array}$ & $\begin{array}{l}\text { - } \mathrm{HbA}_{1 \mathrm{c}} \\
\text { - Weight and height measurements } \\
\text { - Blood pressure reading } \\
\text { - } \text { Adult Literacy in Medicine-Short } \\
\text { - } \text { Form (REALM-SF) } \\
\text { - Spoken Knowledge in Low Literacy } \\
\text { in Diabetes Scale (SKILL-D) } \\
\text { - Diabetes Self-Care Activities } \\
\text { Measure (SDSCA). }\end{array}$ & $\begin{array}{l}\text { - Increased self-efficacy in } \\
\text { patients' self-management } \\
\text { - Increased patients' daily } \\
\text { physical activity }\end{array}$ & $\begin{array}{l}\text { The study only evaluated } \\
\text { short-term outcomes, which } \\
\text { may not reflect long-term } \\
\text { effects (if any). }\end{array}$ \\
\hline 10. & $\begin{array}{l}2010 / \\
\text { Chang et al. }\end{array}$ & $\begin{array}{l}\text { - Exploratory study. } \\
\text { - Five different } \\
\text { routes in Tech } \\
\text { building. }\end{array}$ & $\begin{array}{l}\text { Total sample } \\
\quad=6 \\
\text { Patients }\end{array}$ & $\begin{array}{l}\text { - Passive near- } \\
\text { field radio- } \\
\text { frequency } \\
\text { identification } \\
\text { (RFID) tags. } \\
\text { - Scanning } \\
\text { (personal } \\
\text { digital } \\
\text { assistant) } \\
\text { PDAs. } \\
\\
\text { (Cognitive } \\
\text { impairments) }\end{array}$ & $\begin{array}{l}\text { - Hart and Staveland's NASA task } \\
\text { load index (TLX). }\end{array}$ & $\begin{array}{l}\text { - A simple and effective RFID } \\
\text { based system for indoor way } \\
\text { finding } \\
\text { - Low initial reservations and } \\
\text { resistance users exhibited } \\
\text { about device and technology }\end{array}$ & $\begin{array}{l}\text { - Too small number of } \\
\text { participants. } \\
\text { - PDAs were fragile and not } \\
\text { weather-proof. Protective } \\
\text { measures needed to be } \\
\text { taken to ensure good } \\
\text { maintenance. }\end{array}$ \\
\hline 11. & $\begin{array}{l}2012 / \\
\text { Shing et al. }\end{array}$ & $\begin{array}{l}\text { - Cross-sectional } \\
\text { study. } \\
\text { - Respondents' } \\
\text { household }\end{array}$ & $\begin{array}{c}\text { N/A } \\
\text { Patients }\end{array}$ & $\begin{array}{l}\text { Telecare service } \\
\text { network } \\
\text { (Aging \& chronic } \\
\text { diseases) }\end{array}$ & $\begin{array}{l}\text { - Simple Object Access Protocol } \\
\text { (SOAP) }\end{array}$ & $\begin{array}{l}\text { Familiarisation with preventive } \\
\text { healthcare services at home }\end{array}$ & $\begin{array}{l}\text { The network can be widely } \\
\text { used at home/community } \\
\text { settings. }\end{array}$ \\
\hline 12. & $\begin{array}{l}2009 / \\
\text { Mac- } \\
\text { Culloch et } \\
\text { al. }\end{array}$ & $\begin{array}{l}\text { - Cross sectional } \\
\text { study } \\
\text { - Hospital for sick } \\
\text { children } \\
\text { (SickKids) in } \\
\text { Toronto } \\
\end{array}$ & $\begin{array}{l}\text { Total sample } \\
\quad=11 \\
\text { Patients }\end{array}$ & $\begin{array}{l}\text { Web-based } \\
\text { interaction } \\
\text { (Scoliosis) }\end{array}$ & $\begin{array}{l}\text { - Audio-recorded on focus groups } \\
\text { - Telephone interviews }\end{array}$ & $\begin{array}{l}\text { Uncover social support needs of } \\
\text { the patients }\end{array}$ & $\begin{array}{l}\text { Majority of patients wished to } \\
\text { hear stories from perspectives } \\
\text { of other patients before } \\
\text { deciding on surgical } \\
\text { intervention. }\end{array}$ \\
\hline 13. & $\begin{array}{l}2009 / \\
\text { Sobel et al. }\end{array}$ & $\begin{array}{l}\text { - Cross-sectional } \\
\text { study } \\
\text { - Three diverse } \\
\text { communities in } \\
\text { Chicago }\end{array}$ & $\begin{array}{c}\text { Total sample = } \\
130 \\
\text { Patients }\end{array}$ & $\begin{array}{l}\text { Asthma 1-2-3 } \\
\text { multimedia } \\
\text { curriculum } \\
\text { (Asthma) }\end{array}$ & $\begin{array}{l}\text { Rapid Estimate of Adult Literacy in } \\
\text { Medicine (REALM) }\end{array}$ & $\begin{array}{l}\text { - Improved in asthma } \\
\text { knowledge } \\
\text { - Improvement in health } \\
\text { promoting behaviours }\end{array}$ & $\begin{array}{l}\text { This study encouraged adults } \\
\text { to gain more knowledge on } \\
\text { asthma. }\end{array}$ \\
\hline
\end{tabular}

${ }^{*} N / A=$ Not available 


\begin{tabular}{|c|c|c|c|c|c|c|c|}
\hline No. & $\begin{array}{c}\text { Year / } \\
\text { Author(s) }\end{array}$ & $\begin{array}{c}\text { Study Design } \\
\& \\
\text { Study Setting } \\
\end{array}$ & $\begin{array}{c}\text { Sample Size } \\
(n) / \\
\text { Respondent } \\
\text { Type } \\
\end{array}$ & $\begin{array}{l}\text { Multimedia } \\
\text { applications / } \\
\text { appliances } \\
\text { (conditions) } \\
\end{array}$ & $\begin{array}{l}\text { Instruments / } \\
\text { Measurements }\end{array}$ & Psychosocial Outcomes & Comments \\
\hline 14. & $\begin{array}{l}2009 / \\
\text { Übeyli }\end{array}$ & $\begin{array}{l}\text { - Cross-sectional } \\
\text { study. } \\
\text { - N/A }\end{array}$ & $\begin{array}{c}\text { N/A } \\
\text { Patients }\end{array}$ & $\begin{array}{l}\text { Web-based model } \\
\text { programme } \\
\text { (Diabetes) }\end{array}$ & N/A & $\begin{array}{l}\text { - Maintaining social support } \\
\text { on patients } \\
\text { - Reducing health service } \\
\text { costs on patients }\end{array}$ & $\begin{array}{l}\text { Encouraged interaction } \\
\text { between physicians and } \\
\text { patients. }\end{array}$ \\
\hline 15. & $\begin{array}{l}2008 \text { / } \\
\text { Mosnaim } \\
\text { et al. }\end{array}$ & $\begin{array}{l}\text { - } \text { Randomized } \\
\text { controlled study } \\
\text { - N/A }\end{array}$ & $\begin{array}{l}\text { Total sample } \\
\quad=28 \\
\text { Patients }\end{array}$ & $\begin{array}{l}\text { Music tracks plus: } \\
\text { - Celebrity asthma } \\
\text { health messages } \\
\text { - General health } \\
\text { messages } \\
\text { (Asthma) }\end{array}$ & ZAP Asthma Knowledge & $\begin{array}{l}\text { - Sustained behaviour } \\
\text { changes in patients } \\
\text { - Increased asthma } \\
\text { knowledge. harges in } \\
\text { - Reduced charges } \\
\text { receiving health messages }\end{array}$ & $\begin{array}{l}\text { The study focused on African- } \\
\text { Americans only. }\end{array}$ \\
\hline 16. & $\begin{array}{l}2004 / \\
\text { Diefenbach } \\
\text { \& Butz }\end{array}$ & $\begin{array}{l}\text { - Randomized } \\
\text { controlled study } \\
\text { - At home / at work } \\
\text { / at library }\end{array}$ & $\begin{array}{l}\text { Total sample } \\
\quad=33 \\
\text { Patients \& } \\
\text { Caregivers }\end{array}$ & $\begin{array}{l}\text { Prostate interactive } \\
\text { education system } \\
\text { (PIES). } \\
\text { (Cancer) }\end{array}$ & Interviewed on focus groups of PIES & $\begin{array}{l}\text { - Sustained behaviour } \\
\text { changes in patients } \\
\text { - Minimized the costs to be } \\
\text { spent on health education } \\
\text { programme }\end{array}$ & $\begin{array}{l}\text { Additional patient-education } \\
\text { tool as emotional support post- } \\
\text { surgery. }\end{array}$ \\
\hline 17. & $\begin{array}{c}2004 \text { / } \\
\text { Bielli et al. }\end{array}$ & $\begin{array}{l}\text { - Randomized } \\
\text { controlled study } \\
\text { - Five hospital units } \\
\text { in Milan }\end{array}$ & $\begin{array}{l}\text { Total sample } \\
\quad=97 \\
\text { Patients }\end{array}$ & $\begin{array}{l}\text { Wireless health } \\
\text { outcomes } \\
\text { monitoring system } \\
\text { (WHOMS) } \\
\text { (Cancer) } \\
\end{array}$ & $\begin{array}{l}\text { - Familiarity with communication } \\
\text { technology (FCT) }\end{array}$ & Improvement in patients' QoL. & $\begin{array}{l}\text { Can reduce barriers between } \\
\text { patients and doctors. }\end{array}$ \\
\hline 18. & $\begin{array}{l}2003 / \\
\text { Chambers } \\
\text { et al. }\end{array}$ & $\begin{array}{l}\text { - Exploratory stud. } \\
\text { - Five different } \\
\text { countries: } \\
\text { Northern Ireland, } \\
\text { Republic of Ireland, } \\
\text { England, Portugal and } \\
\text { Sweden }\end{array}$ & $\begin{array}{l}\text { Total sample } \\
\quad=234 \\
\text { Caregivers }\end{array}$ & $\begin{array}{c}\text { Assisting carers } \\
\text { using telematics } \\
\text { interventions to } \\
\text { meet older person } \\
\text { needs (ACTION) } \\
\quad \text { project } \\
\text { (Caregiving) }\end{array}$ & $\begin{array}{l}\text { - Need assessments } \\
\text { - Design development-in-depth } \\
\text { interviews } \\
\text { - Website analysis and measurement } \\
\text { inventory (WAMMI) }\end{array}$ & $\begin{array}{l}\text { Family caregivers became well } \\
\text { prepared to cope with problems } \\
\text { during caregiving. }\end{array}$ & $\begin{array}{l}\text { The programme gives support } \\
\text { to elderly's caregiver and } \\
\text { helps them to prepare for } \\
\text { emergency cases. }\end{array}$ \\
\hline 19. & $\begin{array}{c}2002 \text { / } \\
\text { Nebel et al. }\end{array}$ & $\begin{array}{l}\text { - Randomized } \\
\text { controlled study } \\
\text { - Three German } \\
\text { diabetes centres }\end{array}$ & $\begin{array}{l}\text { Total sample } \\
\quad=126 \\
\text { Patients }\end{array}$ & $\begin{array}{l}\text { Computer-based } \\
\text { interactive diabetes } \\
\text { education } \\
\text { (Diabetes) }\end{array}$ & N/A & $\begin{array}{l}\text { Improved patients' skill to } \\
\text { manage daily nutrition plan. }\end{array}$ & $\begin{array}{l}\text { This program was only to } \\
\text { support the patients. }\end{array}$ \\
\hline 20. & $\begin{array}{l}2002 / \\
\text { Valdez et } \\
\text { al. }\end{array}$ & $\begin{array}{l}\text { - Randomized } \\
\text { controlled study } \\
\text { - Six clinics in } \\
\text { California }\end{array}$ & $\begin{array}{l}\text { Total sample } \\
\quad=1,197 \\
\text { Patients }\end{array}$ & $\begin{array}{c}\text { Multimedia breast } \\
\text { cancer education } \\
\text { (Cancer) }\end{array}$ & $\begin{array}{l}\text { - Breast cancer knowledge } \\
\text { - Attitudes and beliefs with } \\
\text { mammogram status } \\
\text { - Intentions with mammography status }\end{array}$ & $\begin{array}{l}\text { - Improved in knowledge and } \\
\text { attitudes towards breast } \\
\text { cancer } \\
\text { - Reduced the cost to search } \\
\text { health information } \\
\end{array}$ & $\begin{array}{l}\text { The intervention was not able } \\
\text { to alter the minority's fear of } \\
\text { mammography. }\end{array}$ \\
\hline
\end{tabular}

*N/A = Not available 


\section{Discussion}

\section{Review summary}

This review summarized the evidence of existing multimedia-based health education applications, which identified 20 published scientific journal articles. Generally, several types of educational programmes and implementation strategies were built for improving the quality of healthcare [36]. These programmes have been shown to enhance knowledge about certain conditions and to promote positive attitudes from the respondents [3]. As expected, modern technologies can actually help to improve QoL for both patients and their caregivers because the modules facilitated their support within the community [11, 37].

\section{Socio-demographic characteristics}

The fact that $80 \%$ of the studies were carried out in Western countries demonstrated that technology-based health education studies in other countries especially in the Asian region are still lacking. This phenomenon might be due to limited technology sources or the different trends of diseases within the country. Some personnel may also be reluctant to be engaged in such research because of lack of confidence to use such technology and sometimes lack of knowledge about certain diseases [38]. These outcomes certainly did not fully represent the global population which therefore suggest further investigations should be embarked in developing and poorer countries.

Females were the predominant gender in most studies which had led to the overall sample composition being slightly imbalanced (one article even specified females as its only respondents). Otherwise, the gender distributions were mostly equal in the total population. As for the sample size, the extremely small recruitment number from a study could have led to bias in representation, but this could also be due to the rarity of the condition studied i.e. cognitive disabilities.

A mixture of age groups was involved in parallel with the various diseases and different respondent groups. According to the analysis, the respondents were among children, teenagers, adults and even the elderly. All respondents from the groups of children and teenagers were either inpatients or outpatients who received their treatment at the hospital, whereas most of the caregivers were adults. The implemented programmes were advantageous to a wide range of targeted respondents which in turn allowed the determination of the multimedia's suitability across all age groups.

\section{Variations in acceptance by age sub-groups}

Although evidence of ICT benefits have been found in varied populations, the equivalent effects of similar health education programmes across age sub-groups remain unknown [24]. Clearly, children were generally more excited in being engaged with the multimedia-based programmes. It has been hypothesised that this age group was able to extend beyond their existing knowledge to a more sophisticated level of understanding if given the appropriate guidance and instructions [2].

As for the adults, several 'higher level' ICT technologies such as web-, SMS-, mobile- and computerised-based applications were considered acceptable [27]. This was especially pertinent since ICT is increasingly becoming part of their routine communication tools for basic tasks such as checking emails, browsing the websites and sending text messages - a familiarisation process which thus impose little difficulties in adoption and utilisation [39]. As for the elderly, limited scientific evidence can be found on the technology's acceptance since investigations directly involving this age group are generally scarce. If any, studies were mainly concerned with family caregivers assisting their elderly patients to maintain their independence through some formulated interventions [38].

Education programmes with multimedia concepts are undoubtedly more effective in attracting the younger generation (18-29 years old), a trend which should be beneficially maximised in healthcare. They are the most frequently updated with the latest technologies, and those tech-savvy ones usually come from this subset. College students are especially 
acquainted with emails, the internet, cell phones and other 'gizmos' [39]. It is therefore common for them to willingly follow the constantlyevolving technologies and make adjustments in their lives in order to capture optimal benefits from these changes. For example, the 'autonomous indoor wayfinding concept' can stimulate their interest in learning to cope with their cognitive impairment [29].

\section{Multimedia applications / appliances}

Overall, applications based on computer software were the most frequent mode for educational input. The contents of the programme developed through software were usually produced in a form of digital versatile disc (DVD). Through the combination of audiovisual elements (video, icons and simple graphics), a form of persuasive communication was developed as a tool to promote HRQoL among patients or caregivers [22, 40, 41]. Hence, the respondents can access the information for better understanding at home. In addition, the interactive presentation may have provided enjoyment and excitement in learning, hence avoiding boredom. These comprehensive applications were effective in engaging respondents and in improving their health and well-being regardless of the mode of multimedia since all studies demonstrated positive effects on their behavioural activities. Interestingly, when effective and coordinated care was received, the needs for hospital visits were significantly reduced [37].

Computerized programmes could also reduce liability claims by providing health information which helps in offering alternatives and selfmanagement strategies. Besides, it also assists in promoting realistic benefits with regard to medical treatment [42]. Numerous studies have also revealed significant differences between web-based, computer software, telematic and mobile gadgets mechanism. Among these, webbased mode is the most frequently used because online resources hosted at websites are easier to access anytime and anywhere with much cheaper cost than other multimedia mechanisms [39].

\section{Psychosocial outcomes and healthcare cost}

In the context of health research, "psychosocial" can be referred as the combination of intrapersonal, interpersonal and social experiences / interactions which can influence an individual's mind or behaviour [43, 44]. Linking the psychological attributes within environmental condition is important in determining the result of socialisation process because it is the key concept of individual daily activities performances [45-47]. In today's era of high technology, multimedia-based applications or appliances offer strong support in the prevention and detection of diseases, in managing the daily life routine, in connecting the social needs and in increasing the self-efficacy on self-management [48]. Combining telecommunication technology with medical education and personal health promotion provides personal health care to both patients or their caregivers and enhanced well-being [49].

From the views of both patients and caregivers, many generally showed their satisfaction towards the applications which later increased their selfefficacy in many aspects of managing the illnesses. Intrinsically, it was suggested that improvement in self-efficacy could literally increase their level of QoL which was further linked to reduction in patients' clinical symptoms and caregivers' stress. Exposure to the multimedia applications showed an increase in the respondents' anticipation in seeking information about a particular disease, hence stimulating positive attitudes and knowledge improvement.

In addition, it is well known that many patients or their families would face monetary restriction in managing the income spent on patients' treatment, medication and travelling cost. Due to such burdening costs, a distant learning approach would be the most appropriate method to obtain information and even consultation. Several applications met telemedicine goals whereby the technology provided should lessen the fees for health services [48]. Moreover, those studies concentrated on low-income or socioeconomically-disadvantaged respondents in order to identify the external barriers such as 
healthcare cost. For instance, the message contents in the device programmed for asthma patients were delivered by celebrities who were well-received by the community for no monetary charges, further minimising the charges involved [23]. In a diabetes study, the application targeted on early detection which could lead to a considerable cost suppression for subsequent therapeutic measures [21].

\section{Implementation strategies and future trends of ICT in health education}

The appealing, interactive nature of many computerised programmes is capable of encouraging users to engage with the contents long enough to be 'processed' and sustained. [50]. Realizing the full potential of the ICT will require constant commitment to theory-driven research at basic, methodology, clinical, and applied levels [51]. Given such opportunities, a conceptual framework is needed to broaden the scope and boundaries between technology, theory and its applications in healthcare education [52]. In relation to this, training becomes as a major determinant of ICT adoption especially by the healthcare professionals to influence the integration of technologies into clinical practices [53].

In parallel with ICT's popularity, its usage have been widely implemented in current healthcare settings including in Malaysia, precisely as a passive tool to store patient information, patient reminders or prescribing alerts as electronic online health/medical records or computer-based patient records [51]. One way to push forward could be by empowering patients themselves to keep their own health records, for instance in digital forms as 'apps' in smartphones. Of importance also is the facilitation of communication via usage of connectivity (e.g. video conferencing) - which is clearly useful in settings separated by geographical barrier but critically in need of health education or even medical intervention (e.g. surgery). Consequently too, new health educational materials continue to be in demand although free online materials are now readily available. This is not only due to the need for consistent updating (with medical progress), but also because many websites are still not provided with the direct search links - certain programmes are restricted only for their patients and/or families without open access permission to the public, whereby web registration (through healthcare providers) is needed for access [24]. In some sections of the society such as the elderly, those with low literacy, lack of ICT knowledge and rural dwellers, free online materials and utilisation of connectivity may not be their best option. Multi-lingual versions of the material for multi-racial countries like Malaysia and Singapore should be created whereby important cultural values must also be considered and integrated.

In summary, findings from this review have demonstrated the overall benefits of the developed technologies for disseminating relevant health information. Although the applications could not directly improve the patients' clinical conditions, QoL issues and selfmanagement needs for patients and their families have been clearly enhanced.

\section{Limitations}

The main drawback of this review was the limited number of included articles. Our inclusion criteria indicated the requirement of participation among patients, caregivers or combination of both. However, most studies had focused on professional caregivers. Although the studies were conducted worldwide, most were largely conducted in the Western countries (USA) making a global representation impossible. Another limitation is the timerestricted disease distribution as majority of the studies focused on trends of diseases within their country, hence overlooking other types of chronic diseases.

\section{Conclusion}

The overall findings suggest that multimediabased applications are indeed effective for health education in improving awareness, knowledge and psychosocial outcomes for both patients and caregivers. Clearly, these applications show high potential to serve as a support tool to overcome care management problems and to explore 
healthcare needs in the community, particularly for the younger sub-groups. Such convenient technology-based applications have helped in saving cost and time for patients, caregivers as well as physicians in the process of educating, which are ideally be available online and also in various language and culturally-adapted versions. Technology can also foster interactive learning not only for patients' educations but for new generations of public health workers to receive training in innovative communication techniques [51]. Despite the challenges present in using technology as educational material, it has indeed the potential to achieve goals beyond the conservative learning. The promotion of technological solutions to support future health education programmes should be encouraged by health institutions and media. Nevertheless, more research should be conducted in Asia, especially in Malaysia due to the current lack of scientific evidence to support similar benefits for health education in this region.

\section{Acknowledgment}

The authors would like to express their sincere gratitude to Ms Noor Salihah Zakaria, Ms Neni Widiasmoro Selamat, Ms Wan Putri Elena Wan Dali, Ms Norhayati Mustapha and Ms Nurul Diana Dzaraly for their support and guidance.

\section{Reference}

1. Phillips R. The developers handbook to interactive multimedia. A practical guide for educational developers. London: Kogan Page, 1997; (8).

2. McPherson A, Glazebrook C. Double click for health: the role of multimedia in asthma education. Archives of Disease in Childhood 2001; 85:447-449.

3. Schaffer SD, Tian L. Promoting adherence: effects of theory based asthma education. Clinical Nursing Research 2004;13 (1), 38999.

4. Feinstein L, Sabates R, Anderson MT. What are the effects of education on health? Measuring the effects of education on health and civic engagement: Proceedings of the copenhagen symposium 2006.

5. Nolte S, Osborne RH.. A systematic review of outcomes of chronic disease selfmanagement interventions. Quality of Life Research 2013; 22:1805-1816 doi: 10.1007/s11136-012-0302-8.

6. Ofman JJ, Badamgarav E, Henning JM, Knight K, Gano AD Jr, Levan RK. Does disease management improve clinical and economic outcomes in patients with chronic diseases? A systematic review. American Journal of Medicine 2004; 117: 182-192.

7. Sanjaya $M$, Sharma RC. Interactive Multimedia in Education and Training. Indira Gandhi National Open University. Idea Group Publishing, 2004; 434.

8. Titler GM. The Evidence for EvidenceBased Practice. Patient Safety and Quality: An Evidence-Based Handbook for Nurses 2008; (1)

9. Morris M, Lundell J, Dishman E. Catalyzing Social Interaction with Ubiquitous Computing: A needs assessment of elders coping with cognitive decline. Late Breaking Results Paper, Vienna 2004.

10. Mynatt E, Rowan J, Jacobs A, Craighill S. Digital family portraits: supporting peace of mind for extended family members. Proceedings of the Conference on Computer-Human Interaction. New York:ACM 2001; 3(1): 333-340.

11. Shore R. Rethinking the brain. New York: Families and Work Institute 1997.

12. Crosson JC, Heisler M, Subramanian U et al. Physicians' Perceptions of Barriers to Cardiovascular Disease Risk Factor Control among Patients with Diabetes: Results from the Translating Research into Action for Diabetes (TRIAD) Study. Journal of the American Board of Family Medicine 2010; (23):2171-178

13. Pasacreta J, McCorke R. Cancer care: Impact of interventions on caregiver outcomes. In J. Goeppinger (Ed.), Annual Review of Nursing Research (18):127-148. New York: Springer Publishing.

14. Coon DW, Thompson L, Steffen A, Sorocco $\mathrm{K}$. Anger and depression management: 
Psychoeducational skill training interventions for women caregivers of a relative with dementia. Gerontologist 2003; 43(5):678-689.

15. Koop CE: Editorial: A personal role in health care reform. American Journal of Public Health 1995; 85(6):759-760.

16. Shing HL, Wang CY, Lu WH, Lin YY, Yen DC. Design and implementation of a telecare information platform. Journal of Medical Systems 2012; 36:1629-1650 doi: 10.1007/s10916-010-9625-6.

17. Sobel RM, Paasche-Orlow MK, Waite KR, Rittner SS, Wilson EAH, Wolf MS. Asthma 1-2-3: a low literacy multimedia tool to educate African American adults about asthma. Journal of Community Health 2009; 34:321-327 doi: 10.1007/s10900-009-9153-9.

18. Übeyli ED. Medical informatics: a model developed for diabetes education via telemedicine. Journal of Medical System 2009; 33:113-119 doi: 10.1007/s10916-0089171-7.

19. Valdez A, Banerjee $\mathrm{K}$, Ackerson L, Fernandez M. A multimedia breast cancer education intervention for low-income Latinas. Journal of Community Health 2002; 27 (1).

20. Collinge W, Kahn J, Walton T, et al. Touch, caring and cancer: randomized controlled trial of a multimedia caregiver education program. Journal of Supportive Care in Cancer 2013; 21: 1405-1414. doi: 10.1007/s00520-012-1682-6.

21. Diefenbach MA, Butz BP. A multimedia interactive education system for prostate cancer patients: development and preliminary evaluation. Journal of Medical Internet Research 2004; 6 (1), e3. doi: 10.2196/jmir.6.1.e3

22. Khan MA, Shah S, Grudzien A, et al. A diabetes education multimedia program in the waiting room setting. Journal of Diabetes Therapy 2011; 2(3), 178-188. doi: 10.1007/s13300-011-0007-y

23. Mosnaim GS, Cohen MS, Rhoads $\mathrm{CH}$, Rittner SS, Powell LH. Use of MP3 players to increase asthma knowledge in inner-city African-American adolescents. International
Journal of Behavioural Medicine 2008; 15:341-346.

doi: 10.1080/10705500802365656

24. Stanton AL, Morra ME, Diefenbach MA, et al. Responding to a significant recruitment challenge within three nationwide psychoeducational trials for cancer patients. Journal of Cancer Survivorship 2013; 7: 392-403. doi: 10.1007/s11764-013-0282-x.

25. Tancredi DJ, Slee CK, Jerant A, et al. Targeted versus tailored multimedia patient engagement to enhance depression recognition and treatment in primary care: randomized controlled trial protocol for the AMEP2 study. Journal of Health Services 2013; 13, 141. Retrieved from http://www.biomedcentral.com/14726963/13/141.

26. Ahmed S, Bartlett SJ, Ernst P, et al. Effect of a web-based chronic disease management system on asthma control and health-related quality of life: study protocol for a randomized controlled trial. Trials 2011; 12:260 doi: 10.1186/1745-6215-12-260.

27. Antypas K, Wangberg SC. E-rehabilitationan internet and mobile phone based tailored intervention to enhance self-management of cardiovascular disease: study protocol for a randomized controlled trial. Journal of Cardiovascular Disease 2012; 50. Retrieved from http://www.biomedcentral.com/14712261/12/50.

28. Bielli E, Carminati F, La Capra S, Micaela L, Brunelli C, Tamburini M. wireless health outcomes monitoring system (WHOMS): development and field testing with cancer patients using mobile phones. BMC Medical Informatics and Decision Making 2004; 4:7. Retrieved from http://www.biomedcentral.com/14726947/4/7.

29. Chang YJ, Peng SM, Wang TS, Chen SF, Chen YR, Chen, HC. Autonomous indoor wayfinding for individuals with cognitive impairments. Journal of Neuroengineering and Rehabilitation 2010; 7:45. Retrieved from

http://www.jneuroengrehab.com/content/7/1/ 45 
30. Lua PL, Neni WS. Health-related quality of life improvement via telemedicine for epilepsy: printed versus SMS-based education intervention. Quality of Life Research 2013; doi: 10.1007/s11136-0130352-6

31. MacCulloch R, Donaldson S, Nicholas D, et al. Towards an understanding of the information and support needs of surgical adolescent idiopathic scoliosis patients: a qualitative analysis. Scoliosis Journal 2009; 4:12. doi: 10.1186/1748-7161-4-12

32. Meldrum D, Herdman S, Moloney R, et al. Effectiveness of conventional versus virtual reality based vestibular rehabilitation in the treatment of dizziness, gait and balance impairment in adults with unilateral peripheral vestibular loss: a randomised controlled trial. BMC Ear, Nose \& Throat Disorders 2012; 12:3. Retrieved from http://www.biomedcentral.com/14726815/12/3.

33. Nebel IT, Blüher M, Starcke U, Müller UA, Haak T, Paschke R.. Evaluation of a computer based interactive diabetes education program designed to train the estimation of the enrgy or carbohydrate contents of foods. Patient Education and Counseling 2002; 46:55-59. Retrieved from http://www.elsevier.com/locate/pateducou.

34. Spinsante S, Gambi E. Remote health monitoring for elderly through interactive television. BioMedical Engineering Online 2012; 11: $54 . \quad$ Retrieved from http://www.biomedical-engineeringonline.com/content/11/1/54.

35. Chambers MG, Connor SL, McGonigle M, Diver MG. Multimedia software to help caregivers cope. Journal of the American Medical Informatics Association 2003; 10: 504-511. doi: 10.1197/jamia.M1028.

36. McNuly JA, Hoyt A, Gruener G, Chandrasekhar A, Espiritu B, Price RJr, Naheedy R. An analysis of lecture video utilization in undergraduate medical education: associations with performance in the courses. BMC Medical Education 2009; 9 (6).

37. Moeinedin F, Moineddin R, Jadad AR, Hamid JS, To T, Beyene J. Application of biomedical informatics to chronic pediatric diseases: a systematic review. BMC Medical Informatics and Decision Making 2009; 9, 22. doi: 10.1186/1472-6947-9-22.

38. Astell A, Alm N, Gowans G, Ellis M, Dye $\mathrm{R}$, Vaughan P. Involving older people with dementia and their carers in designing computer based support systems - some methodological considerations. Universal Access in the Information Society 2009 (8):49-58.

39. Kittleson MJ. The future of technology in health education: challenging the traditional delivery dogma. American Journal of Health Education 2009; 40 (6). Retrieved from http://opensiuc.lib.siu.edu/phe_pubs/1.

40. Hinyard LJ, Kreuter MW. Using narrative communication as a tool for health behaviour change: a conceptual, theoretical and empirical overview. Health Education Behaviour 2007; 34:777-792.

41. Schwartzberg JG, VanGeest JB, Wang CC. Understanding health literacy. Implications for Medicine and Public Health. Chicago: American Medical Association 2005.

42. Bartlett EE. Patient-centered computing: can it curb malpractice risk?. Retrieved from:http://www.ncbi.nlm.nih.gov/pmc/artic les/PMC2248478/pdf/procascamc000020095.1993.

43. Mosey AC. Applied scientific inquiry in the health professions: An epistemological orientation. American Occupational Therapy Association 1996.

44. Garrard J, Mullen L, Ostrom JJ, McNeill LA, Etzwiller DD. Clinical evaluation of the impact of a patient education program. Diabetes Education 1990; 16:394-400.

45. Castaldini M, Saltmarch M, Luck S, Sucher $\mathrm{K}$. The development and pilot testing of a multimedia CD-ROM for diabetes education. Diabetes Education 1998; 24:285296.

46. Singh-Manoux A, Richards M, Marmot M. Leisure activities and cognitive function in middle age:evidence from the Whitehall II study. Journal of Epidemiology Community Health 2003; 57:907-913. 
47. Güler NF, Übeyli ED. Theory and applications of telemedicine. Journal of Medical Systems 2002; 26(3):199-220.

48. Abrams DB, Mills S, Bulger D. Challenges and future directions for tailored communication research. Annals of Behavioural Medicine, 1999; 21 (4): 299306.

49. Übeyli, ED. Medical informatics: a model developed for diabetes education via telemedicine. Journal of Medical System, 2009; 33, 113-119. doi: 10.1007/s10916008-9171-7.

50. Haux R. Health information systems - past, present, future. International Journal of Medical Informatics 2006; 75, 268-281. doi: 10.1016/j.jimedinf.2005.10.001.

51. Van Achterberg T, Schoonhoven L, Grol R. Nursing implementation science: How evidence-based nursing requires evidencebased implementation. Journal of Nursing Scholarship 2008; 40(4), 302-310.

52. Fors M, Moreno A. The benefits and obstacles of implementing ICTs strategies for development from bottom-up approach. Aslib Proceedings 2002; 54(3), 198-206. doi: 21_2214;11120,/1021--2<-6

53. Gagnon MP, Légaré $F$, Labrecque $M$, et al. Interventions for promoting information and communication technologies adoption in healthcare professionals. Cochrane Database Syst Rev 2009; (1). doi: 10.1002/14651858.CD006093.pub2. 